\title{
Urban modeling for 3D GIS purposes from laser scanning: an implementation for university campus
}

\section{SIGRADI2018 TECHNOPOLITICAS \\ xxii congresso da sociedade iberoamericana de gráfica digital 22th conference of the iberoamerican society of digital graphics 07|08|09|novembro|2018 iau usp | são carlos | sp br}

\author{
Elaine Gomes Vieira de Jesus \\ Universidade Federal da Bahia | Brazil | elainegomes623@gmail.com
}

\author{
Arivaldo Leão de Amorim \\ Universidade Federal da Bahia | Brazil | alamorim@ufba.br
}

Natalie Johanna Groetelaars

Universidade Federal da Bahia | Brazil | natgroet@ufba.br

Vivian de Oliveira Fernandes

Universidade Federal da Bahia | Brazil | vivian.fernandes@ufba.br

\begin{abstract}
3D Geographic Information Systems (3D GIS) are systems capable of performing spatial analyzes that consider a three-dimensional representation of objects, through their planialtimetric coordinates. City Geography Markup Language (CityGML) is used for city and urban applications. The main challenges for this system implementation refer to the techniques used to obtain data, and their formats, in addition to the various software used in geometric modeling. In this way, this article aims to discuss geometric modeling for a university campus using airborne laser scanning data, aiming at the creation of database for applications development.
\end{abstract}

Keywords: 3D GIS; SIG 3D; CityGML; Geometric modeling.

\section{INTRODUÇÃO}

Os Geographic Information Systems (GIS) ou Sistemas de Informações Geográficas (SIG) possuem várias aplicações urbanas e podem estar associados a visualizações 2D, 2,5D, 3D e 4D. Os SIG 2D apresentam objetos bidimensionais e suas respectivas coordenadas correspondentes ao X e Y. Em um SIG 2,5D existe uma informação 3D associada, representada pelo atributo cor a partir de um modelo digital de elevação. O SIG 3D é aquele que possibilita visualizar as representações $\mathrm{X}, \mathrm{Y}$ e Z. Já o SIG 4D permite o estudo temporal de uma área através de variáveis associadas à cronologia numa representação tridimensional.

Os dados e processos a serem representados num GIS são modelados de forma que o Sistema do Mundo Real (SMR) passe por um processo de abstração no qual o analista seleciona as informações necessárias e suficientes para representar o SMR numa determinada escala e para uma dada finalidade.

Algumas pesquisas têm sido desenvolvidas para integrar SIG ao gerenciamento de campus universitários, como por exemplo Bansal (2014) que usou o GIS para o planejamento espacial do campus do Instituto Nacional de Tecnologia (NIT) Hamirpur, Índia. O estudo integrou análises baseadas na localização, atributos semânticos, modelagem digital do terreno (superfície topográfica), topologia das edificações, análises espaciais e não espaciais em uma única plataforma 3D.
As representações 3D para modelos de cidades, segundo Kemec et al. (2009), são formas de comunicação mais eficazes e mais precisas do que as representações $2 \mathrm{D}$, pois reduzem significativamente o esforço cognitivo necessário para interpretar os dados e as análises dos resultados. Essa compreensão auxilia na tomada de decisões de autoridades nacionais, estaduais e municipais, aumentando o nível de eficiência e eficácia das mesmas.

Os modelos geométricos de cidades associam representações geoespaciais rigorosas a uma base de dados alfanumérica, que as aproxima do mundo real, combinando geometria (forma), aparência e semântica. Na modelagem geométrica é necessário definir o Nível de Detalhe (Level of Detail - LOD), que expressa a precisão e a fidedignidade com as quais os elementos devem ser representados e as classes de elementos a serem incluídas (REDWEIK et al., 2007).

Nesse contexto, existem diferenças no uso do termo LOD para GIS e para Computer Graphics. Segundo Biljecki (2017), o LOD de um modelo geométrico de cidade diz respeito ao grau de adesão do mesmo em relação à realidade, enquanto que em Computer Graphics é necessário haver uma simplificação dos dados (processo de generalização) e não considera a semântica como no GIS.

A modelagem geométrica de cidades é um processo que envolve muitas variáveis, especialmente no tocante a: diferentes técnicas de aquisição dos dados, variedade de 
ferramentas para modelagem, dificuldades de interoperabilidade entre eles, além do uso de normas e padrões distintos.

Nesse contexto, o presente artigo discute uma abordagem metodológica para a modelagem geométrica do campus Ondina-Federação da Universidade Federal da Bahia (UFBA). Foi estabelecido que o modelo geométrico construído a partir de dados de varredura a laser aerotransportada seria compatível com LOD 1 do padrão City Geography Markup Language (CityGML), visando a produção de uma base de dados do campus para o desenvolvimento de aplicações 3D GIS.

\subsection{TÉCNICAS PARA OBTENÇÃO DOS DADOS PARA MODELAGEM GEOMÉTRICA DE CIDADES}

Como fonte de dados para modelagem geométrica de cidades, Bobkowska et al. (2017) ressaltam o laser scanning do tipo Light Detection and Ranging (LIDAR) como uma tecnologia adequada à captura de dados, tendo em vista a sua grande precisão e o curto tempo de trabalho em campo.

A varredura a laser é um sistema de captura de dados que permite a obtenção de coordenadas tridimensionais de pontos da superfície terrestre e de objetos sobre ela, a partir da emissão de pulsos de raios laser disparados por um sensor. O processo baseia-se no tempo e na intensidade de emissão/retorno do pulso laser, que permite o cálculo da distância entre o ponto de reflexão do pulso e o sensor. Este sistema permite captar informações sobre o terreno, as edificações, bem como sobre os demais objetos como vegetação, vias, postes etc.

A nuvem de pontos consiste num tipo de representação de dados eficiente e eficaz, na medida em que a partir desta podem ser elaborados Modelos Digitais de Terreno (MDT) e Modelos Digitais de Superfície (MDS). Estes dados podem ser utilizados em projetos voltados para elaboração/atualização de base cartográfica, obtenção da cota Z para criação de modelos geométricos de cidades e várias outras aplicações urbanas.

A produção de modelos digitais do terreno enfrenta um problema que segundo Centeno e Mitishita (2007), consiste em diferenciar as feições que correspondem ao terreno daquelas correspondentes ao que está acima desse como edificações e vegetação. Uma das formas de obter a altura dos objetos que estão acima do terreno é realizar a normalização do Modelo Digital de Superfície, ou seja, calcular a diferença entre o MDS e MDT e assim obter diretamente 0 valor das alturas apenas das edificações.

A escolha da técnica a ser utilizada está relacionada também ao nível de detalhe que se deseja alcançar. Akmaliaa et al. (2014) ressaltam que os dados obtidos pela tecnologia LIDAR aerotransportada podem ser usados para gerar modelos compatíveis com os LOD0, LOD1 e LOD2, enquanto que os dados de fotogrametria de curta distância (ou terrestre) são mais adequados para gerar modelos detalhados em LOD3 e LOD4. Como o objetivo deste trabalho é gerar modelos compatíveis com o LOD 1, foi dada ênfase no uso de dados LIDAR aerotransportado.

\subsection{SOFTWARES USADOS PARA MODELAGEM GEOMÉTRICA DE CIDADES}

$\mathrm{Na}$ literatura são encontrados diversos softwares para modelagem geométrica de cidades. Destacam-se como mais utilizados: Modelur e CityEditor (plugins do Sketchup), CityEngine e Grasshopper (plugin do Rhinoceros).

O SketchUp (Trimble) é um programa para modelagem geométrica, e seu plugin Modelur (Figura 1) possibilita a modelagem parametrizada voltada ao planejamento urbano. Seu principal diferencial é utilizar as modelagens $3 \mathrm{D}$ realizadas dentro do software como referência, atribuindo valores como índices de zoneamento ao mesmo tempo que permite a modelagem paramétrica das edificações (SILVA JUNIOR, 2016).

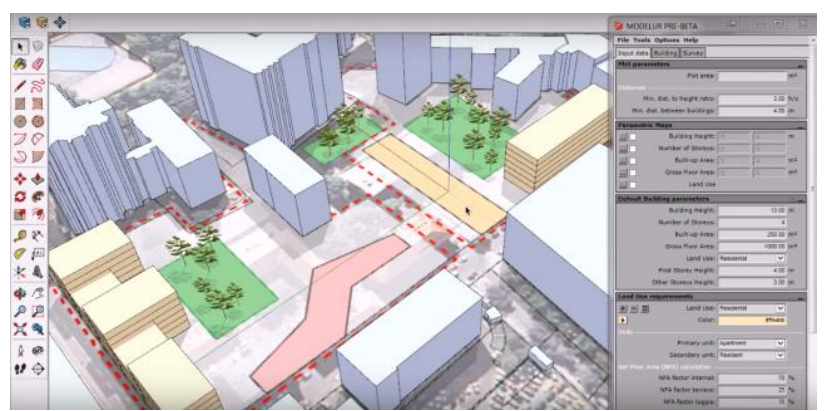

Figura 1: Interface do plugin Modelur. Fonte: autores.

O plugin CityEditor (Figura 2) permite a importação, edição e exportação de modelos CityGML. Esta ferramenta importa: modelos CityGML; modelos de terreno; texturas; dados vetoriais (ponto, linha e polígono) em formato SHP, DXF, GML, JSON, GEOJSON; nuvem de pontos e modelos de superfícies trianguladas de arquivos PLY e OBJ. Possibilita a edição, adição e exclusão de atributos para edifícios e superfícies e a exportação de objetos para modelos CityGML (3DISGmbH, 2016).

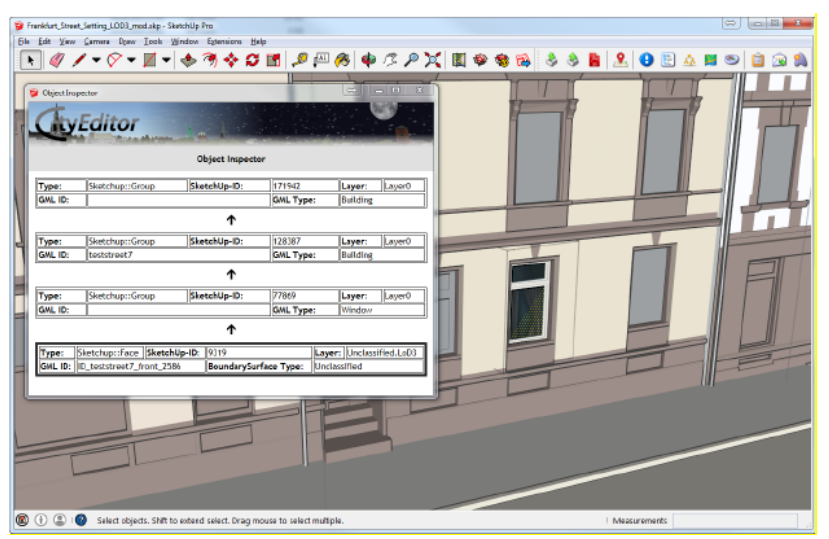

Figura 2: Interface do plugin CityEditor. Fonte: autores.

Já o Grasshopper é um plugin do Rhinoceros (Rhino Software) e tem sido usado para diversas aplicações, inclusive aquelas denominadas de "urbanismo paramétrico". Para modelagem 3D de cidades podem ser utilizados os add-on Heron (Figura 3) e Merkat (que são plugins do Grasshopper) e permitem a importação de dados em formato shapefile (SHP) que possui a integração com GIS e realizam a extrusão dos polígonos através da altura. 


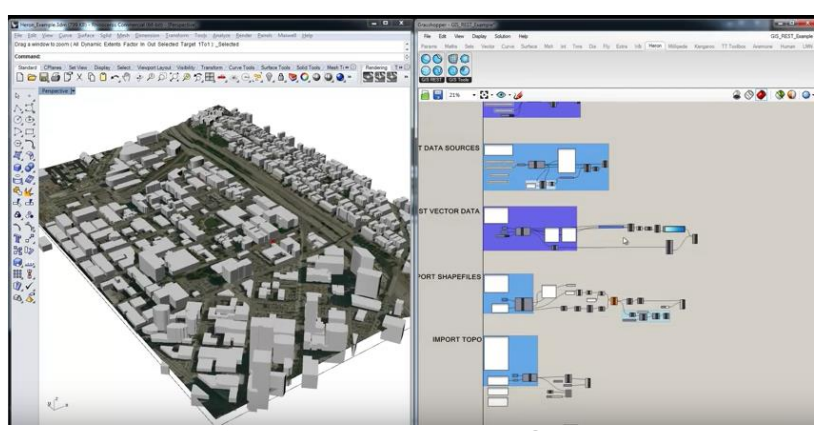

Figura 3: Interface do plugin Heron do Grasshopper. Fonte: autores.

Por usa vez, o CityEngine (ESRI) é um software para modelagem urbana que possui integração com ferramentas GIS e pode ser usado para fins de planejamento, operação e monitoramento. A ferramenta utiliza a linguagem de programação Computer Generated Architecture (CGA) para gerar modelos através de um conjunto de regras pré-definidas. A interface desse software, pode ser visualizada na Figura 4.

O CityEngine destaca-se em relação aos outros dois softwares pelo fato de importar diretamente dados em formato Shapefile (SHP), File Geodatabase (GDB), KML e OpenStreetMap (OSM).

Entretanto, segundo Lima e Freitas (2016), o fato do CityEngine ser um software estrangeiro torna o processo de elaboração de algoritmos e regras paramétricas um pouco complexo, devido à incompatibilidade das "regras prontas" com a lógica da normativa urbanística brasileira.

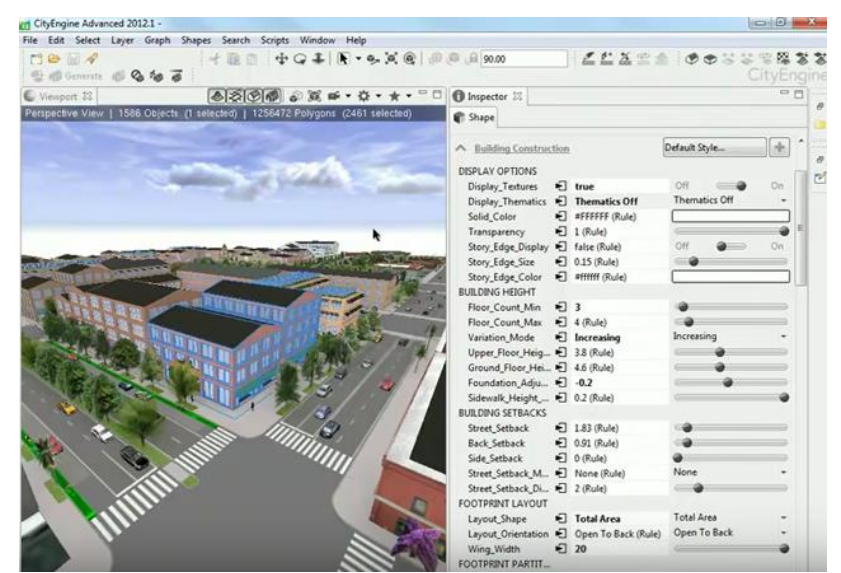

Figura 4: Interface do CityEngine. Fonte: autores.

Entretanto, segundo Zlatanova et al. (2002), verifica-se que os softwares que manipulam objetos 3D apresentam algumas limitações. Ou seja, um software é usado para armazenar dados, outro para visualizar e outro para converter, mas até o momento não foram desenvolvidos softwares GIS 3D totalmente integrados, capazes de estruturar, manipular, analisar, visualizar, representar e converter dados 3D em formatos próprios para modelagem geométrica da cidade.

\subsection{PADRÕES E FORMATOS PARA A MODELAGEM GEOMÉTRICA DE CIDADES}

Em relação aos padrões de dados para modelagem, destaca-se o City Geography Markup Language (CityGML) que é um padrão internacional para um modelo aberto de dados, baseado na linguagem XML, que visa o armazenamento e o desenvolvimento de aplicações que envolvem representações tridimensionais de cidades. Este padrão foi elaborado pelo Special Interest Group 3D (SIG3D), um grupo de trabalho que lida com questões relativas à modelagem, armazenamento, visualização e a manipulação de paisagens e modelos de cidade em 3D.

Em 2008, a primeira versão do CityGML foi submetida ao Open Geospatial Consortium (OGC), organização internacional que visa a criação de padrões de interoperabilidade e qualidade para a comunidade de aplicações geoespaciais. Em 2012, foi aprovada a versão 2.0 e atualmente encontra-se em processo de finalização da versão 3.0 do CityGML, que deverá ser lançada no final de 2018 ou início de 2019 (KUTZNER; KOLBE, 2018). O objetivo do CityGML é alcançar uma definição comum (OpenGIS) das entidades básicas, atributos e relações de um modelo tridimensional de cidade. Os módulos que compõem a versão atual (2.0) do CityGML estão expressos no Quadro 1.

Para Groger e Plumer (2012), o CityGML define a geometria tridimensional, a aparência, a topologia e a semântica dos tipos de objetos mais relevantes em contextos urbanos ou regionais, em contraposição aos modelos puramente geométricos/gráficos como Keyhole Markup Language (KML), Virtual Reality Modeling Language (VRML) e X3D, que não possuem atributos semânticos significativos.

Quanto às características desses formatos, Zlatanova et al (2012) explica que:

- O VRML foi lançado em 1995 e aceito como padrão pelo consórcio Web 3D. Foi concebido como um padrão web para troca de gráficos, proporcionando possibilidades de interação com o mundo 3D. Praticamente é uma linguagem para modelar cenas e interações 3D realistas. Este padrão não é baseado em XML, isso faz com que grandes modelos resultem em arquivos grandes.

- O consórcio Web 3D parou o desenvolvimento do padrão VRML após 1998 e concentrou-se no formato de arquivo X3D baseado em XML. Este formato de arquivo é um aprimoramento do VRML, apesar de ainda ser menos usado que esse. Entretanto dos poucos softwares disponíveis para visualização de dados na Internet quase nenhum permite exportação e importação de X3D.

- O KML é um formato XML para anotação geográfica e visualização de objetos dentro do Google Maps e Google Earth. O KML foi desenvolvido pela empresa Keyhole, Inc., que foi então adquirida pelo Google. Naquela época, o KML foi adotado como padrão pelo OGC. O suporte da geometria no KML está alinhado com o GML. O KML é adequado para aplicativos da Web e amplamente aceito, mas as geometrias são representadas sem semântica.

O CityGML suporta diferentes níveis de detalhe e estes podem variar do LOD0 ao LOD4. No LOD0, o modelo representa apenas a superfície do terreno, na escala da 
cidade; no LOD1, o perímetro e a altura das edificações são representados por extrusão dos poliedros; no LOD 2 , as características das edificações como formato do telhado e texturas estão presentes; no LOD 3, representa-se a escala da edificação, na qual verifica-se um melhor detalhamento da parte externa da mesma, com modelagem de saliências e outros detalhes; e no LOD 4, representa-se a parte interna das edificações, como divisões internas e seus equipamentos com riqueza de detalhes. Essa representação pode ser vista na Figura 5.

Quadro 1: Módulos e campos temáticos do CityGML

\begin{tabular}{|c|c|}
\hline Módulos & Descrição \\
\hline CityGML Core & O módulo Núcleo define os componentes básicos do modelo de dados do padrão CityGML. \\
\hline Appearance & $\begin{array}{l}\text { O módulo Aparência fornece os recursos para modelar a aparência dos objetos CityGML, ou seja, as } \\
\text { propriedades visuais da superfície dos objetos. }\end{array}$ \\
\hline Bridge & $\begin{array}{l}\text { O módulo Ponte permite a representação de aspectos espaciais e temáticos das pontes e seus } \\
\text { componentes, em quatro níveis de detalhe (LOD 1-4). }\end{array}$ \\
\hline Building & $\begin{array}{l}\text { O módulo Edificações permite representar aspectos espaciais e temáticos das edificações, e seus } \\
\text { componentes, em cinco níveis de detalhe (LOD } 0-4) \text {. }\end{array}$ \\
\hline CityFurniture & O módulo Mobiliário Urbano é usado para representar o mobiliário urbano das cidades. \\
\hline CityObjectGroup & $\begin{array}{l}\text { O módulo Grupo de Objetos da Cidade permite definir grupos de objetos diversos, que constituem um } \\
\text { modelo parcial da cidade. }\end{array}$ \\
\hline Generics & $\begin{array}{l}\text { O módulo Genérico fornece extensões genéricas para o modelo de dados CityGML, que podem ser } \\
\text { usadas para modelar características não abrangidas pelas classes temáticas predefinidas do padrão } \\
\text { CityGML. }\end{array}$ \\
\hline LandUse & $\begin{array}{l}\text { O modulo Uso da Terra permite a representação de áreas da superfície da terra dedicadas a um uso da } \\
\text { terra específico. }\end{array}$ \\
\hline Relief & O módulo Relevo permite a representação do terreno num modelo de cidade. \\
\hline Transportation & $\begin{array}{l}\text { O módulo Transportes é usado para representar as características de transportes da cidade, por exemplo, } \\
\text { vias, caminhos, ferrovias, ou praças. }\end{array}$ \\
\hline Tunnel & $\begin{array}{l}\text { O módulo Túnel facilita a representação dos aspectos espaciais e temáticos dos túneis e os seus } \\
\text { componentes, em quatro níveis de detalhe (LOD 1-4). }\end{array}$ \\
\hline Vegetation & $\begin{array}{l}\text { O módulo Vegetação fornece as classes temáticas para representar os objetos da vegetação, isolados ou } \\
\text { contínuos. }\end{array}$ \\
\hline WaterBody & $\begin{array}{l}\text { O módulo Corpos D’água representa a geometria 3D e os aspectos temáticos dos rios, canais, lagos e } \\
\text { bacias. }\end{array}$ \\
\hline TexturedSurface & $\begin{array}{l}\text { O módulo Superfície Texturizada permite a atribuição de propriedades visuais aparência e texturas para } \\
\text { as superfícies dos objetos 3D. A informação aparência fornecida por este módulo pode ser suprida o } \\
\text { módulo Aparência sem prejuízos. }\end{array}$ \\
\hline
\end{tabular}

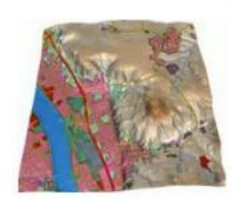

LOD0

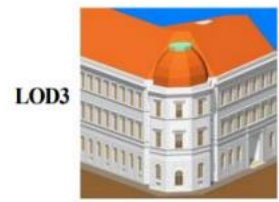

LOD1

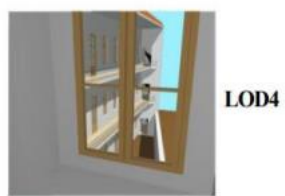

Figura 5: Os cinco níveis de detalhes Fonte: GRÖGER et al., 2012.

\subsection{EXEMPLOS DE ESTUDOS E PROTÓTIPOS CONSTRUÍDOS PARA MODELAGEM 3D}

Siret et al. (2006), desenvolveram um protótipo de 3D GIS capaz de manipular entidades tridimensionais (representando edifícios) e de associar informações como simulação da radiação solar luminosa e térmica, simulação de fluxos de vento, e simulação de níveis sonoros. Este GIS usou como modelo de tipo geométrico o B-REP que apresenta um conjunto de faces poligonais planas, com uma descrição topológica associada. Com esse protótipo, eles concluíram que a modelagem 3D constitui um recurso adequado à gestão das cidades em termos de reabilitação, renovação urbana ou expansão urbana.

Muitas pesquisas estão sendo desenvolvidas pela equipe do laboratório Map-Page do Instituto Nacional de Ciências Aplicadas (INSA) de Strasbourg na França relacionadas à modelagem 3D. Um dos trabalhos abordados por Koehl et al. (2008) envolveu um levantamento topográfico e fotogramétrico do Lycée International des Pontonniers de Strasbourg (uma escola do ensino médio). Os dados utilizados para modelagem tridimensional foram adquiridos com a técnica de fotogrametria terrestre. A modelagem geométrica das edificações foi realizada no software Sketchup Pro permitindo a combinação do modelo com as texturas, mantendo representações fiéis aos edifícios. Os modelos foram exportados em formato VRML, que permite a gravação das texturas associadas aos edifícios e a visualização de cenas $3 \mathrm{D}$ na web é realizada através de um navegador de internet. Por fim, foi construído um 3D GIS para representar estes modelos, utilizando servidor Apache, SGBD MySQL e linguagem de programação PHP.

Outro estudo de modelagem 3D de cidades foi desenvolvido por Julin et al. (2018) para seis das maiores cidades da Finlândia: Helsínquie, Espoo, Tampere, Vantaa, Turku e Oulu. Este estudo inclui informações sobre as plataformas usadas na modelagem da cidade, bem como os softwares de visualização, os níveis de 
detalhe do modelo e indicam se os mesmos estão disponíveis para visualização.

Dentre estes modelos 3D de cidades da Finlândia, destaca-se o de Helsínquie, pois desde a década de 1980 vinham sendo desenvolvidos modelos geométricos da mesma, culminando com o lançamento do modelo semântico e tridimensional de cidade, baseado no padrão CityGML. Para a construção da geometria, foram usados dados de varredura a laser aerotransportada e fotografias aéreas. Os dados utilizados na construção do modelo 3D, compatível com o LOD 2, do CityGML estão disponíveis no portal de informações públicas de Helsinque [Helsinki Region Infoshare (HRI) https://hri.fi/data/en_GB/dataset].

Groger e Plumer (2012) apresentam modelos 3D de cidades europeias. Na Alemanha, muitos municípios possuem modelos compatíveis com LOD 2, sendo que os modelos de Berlim, Colônia, Dresden, Dusseldorf e Munique são compatíveis com o LOD 3.

Ainda segundo esses autores, cidades como Mônaco, Genebra, Zurique, Leeuwarden e algumas cidades na Dinamarca usam o padrão CityGML para modelagem em LOD 2 ou 3. Por fim, na Ásia também existem modelos de cidade no padrão CityGML: Istambul (Turquia) com LOD 1 e 2; Yokohama (Japão) em LOD2, e Doha (Katar) com LOD 3.

De acordo com Biljecki (2017), muitos governos locais utilizam modelos tridimensionais de cidades em aplicações de planejamento urbano e simulações ambientais visando: investigar como o ruído do tráfego se propaga no espaço urbano, estimar sombras projetadas pelos edifícios, e calcular a quantidade de irradiação solar sobre o telhado das edificações a fim de avaliar se é economicamente viável instalar painéis fotovoltaicos, dentre outras aplicações.

\section{METODOLOGIA}

Para realização deste trabalho foram utilizados os seguintes recursos:

- Mapa base do Sistema Cartográfico da Região Metropolitana de Salvador (SICAR, 1992) em escala 1:2000; e Sistema Cartográfico e Cadastral do Município de Salvador (SICAD, 2006) em escala 1:2000, dos quais foram extraídos os perímetros das edificações (footprint);

- Perímetros das edificações do campus atualizados no ano de 2017, por meio de imagens obtidas através de mapeamento com Veículo Aéreo Não-Tripulado (VANT) realizada no âmbito do DETG-UFBA, por Elias (2017);

- Dados semânticos sobre as edificações do campus extraídos dos websites das unidades universitárias da UFBA;

- Nuvem de pontos (com aproximadamente 10 pontos $/ \mathrm{m}^{2}$ ), Modelo Digital de Terreno (MDT), Modelo Digital de Superfície (MDS) e Ortofoto provenientes de varredura a Laser aerotransportada;
- Complementos do software QGIS: "Point Sampling Tools" usado para atribuir aos centroides das bases das edificações os valores das alturas das mesmas, correspondentes à diferença entre o MDS e MDT no ponto considerado, e "Qgis2ThreeJS" para a extrusão dos perímetros das edificações;

Inicialmente foi realizada a compilação dos dados para construção do mapa base, ou seja, foram reunidas as bases de dados vetoriais (SICAR, 1992), SICAD (2006) e Elias (2017). Foi verificada a localização dos perímetros das edificações e realizada a vetorização de algumas edificações que não constavam na base de dados, através das ortofotos, no software QGIS.

Em seguida, foram gerados os centroides para os perímetros de cada edificação e aos centroides foram atribuídos o valor da altura do terreno e das edificações com base respectivamente no MDT e MDS através do complemento "point sampling tools" do QGIS.

Para determinação da altura das edificações foi realizada a normalização do MDS, calculando a diferença de altitude do MDS em relação ao MDT permitindo a obtenção das alturas médias das edificações.

Posteriormente foi realizada a união dos perímetros das edificações, com a altura média atribuída aos centroides e com dados semânticos em formato CSV, contendo os seguintes atributos: nome da unidade universitária, área de conhecimento, cursos de graduação, cursos de pósgraduação e número de salas de aula.

Por fim, foi realizada a modelagem geométrica das edificações no QGIS usando o complemento Qgis2ThreeJS. A técnica utilizada permite a extrusão dos perímetros das edificações com base na altura média das mesmas e possibilita agregar semântica aos objetos.

A modelagem foi realizada no software QGIS, pois a construção do mapa base foi realizada nesta plataforma e devido aos problemas comuns de interoperabilidade para importação/exportação em diferentes formatos de dados para realizar a modelagem em outro software.

\section{RESULTADOS}

A área de estudo representa o campus OndinaFederação da Universidade Federal da Bahia em Salvador na Bahia. O campus abrange uma área de 60 ha, contendo 75 edificações. A Figura 6 mostra a localização da área de estudo.
5 


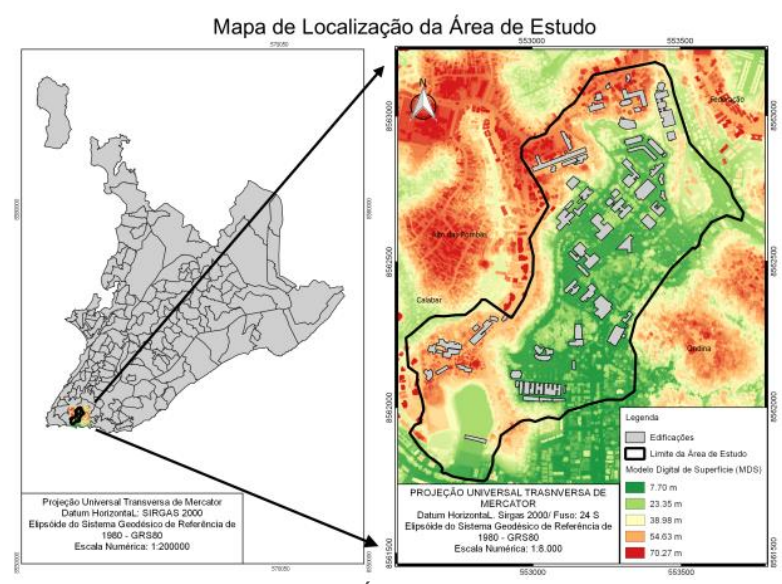

Figura 6: Localização da Área de Estudo. Fonte: autores.

A construção do mapa base permitiu identificar que a área possui 75 edificações (conforme Figura 7), que agregam:

- 15 unidades universitárias;

- 6 pavilhões de aulas;

- 2 restaurantes universitários;

- 2 bibliotecas;

- 2 bancos;

As unidades universitárias são segundo o Estatuto e Regimento da UFBA (2010), órgãos de execução das atividades acadêmicas e de lotação de pessoal docente e técnico-administrativo. Estas unidades compreendem duas modalidades: Faculdades ou Escolas que são unidades de ensino, pesquisa e extensão definidas por sua missão de formação em carreiras acadêmicas, profissionais, tecnológicas e artísticas; e Institutos, unidades de ensino, pesquisa e extensão definidas por sua missão de formação acadêmica em campos científicos gerais ou áreas de conhecimento disciplinares, multidisciplinares e interdisciplinares.

Os demais prédios representam anexos das unidades universitárias e prédios onde são exercidas funções administrativas.

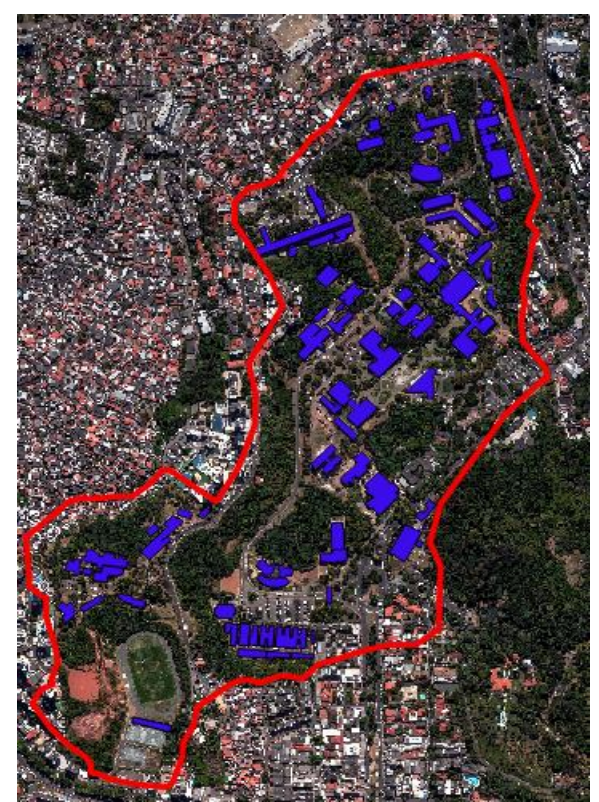

Figura 7: Edificações da Área de Estudo. Fonte: autores.
Percebe-se também que este campus, por ser o maior da Universidade Federal da Bahia, apresenta também um maior número de cursos de graduação e pós-graduação. Sendo 57 cursos de graduação e 48 cursos de pósgraduação distribuídos nas unidades universitárias, como pode ser visto nas Figuras 8 e 9.

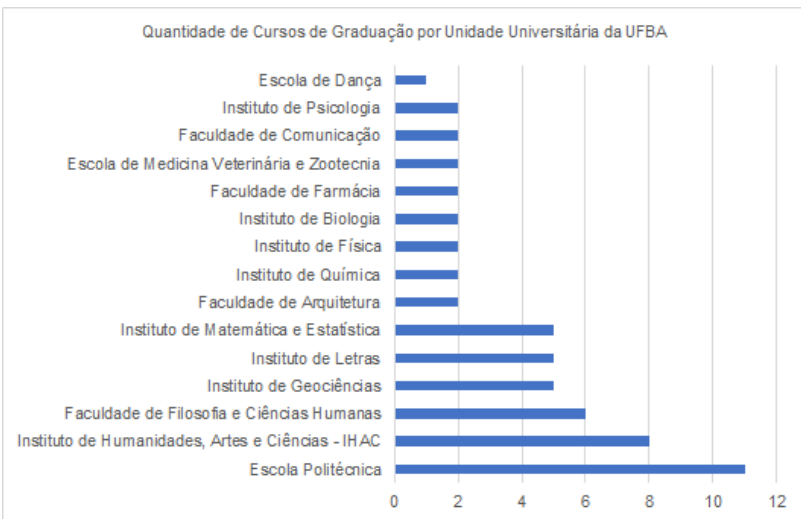

Figura 8: Número de cursos de graduação por Unidade Universitária. Fonte: autores.

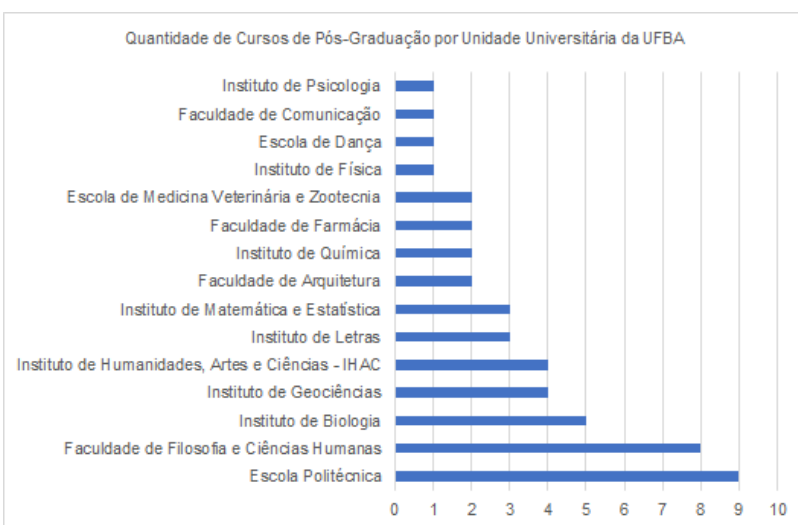

Figura 9: Número de cursos de pós-graduação por Unidade Universitária. Fonte: autores.

A modelagem geométrica realizada através do complemento Qgis2ThreeJS no QGIS demonstrou que o terreno apresenta altitudes variando de 8 a $59 \mathrm{~m}$, e as edificações chegam a $30 \mathrm{~m}$ de altura. Também foi possível verificar que a modelagem atende aos requisitos do modelo CityGML para LOD 1 permitindo identificar informações semânticas (Figura 10) e agregando geometria, aparência e topologia.

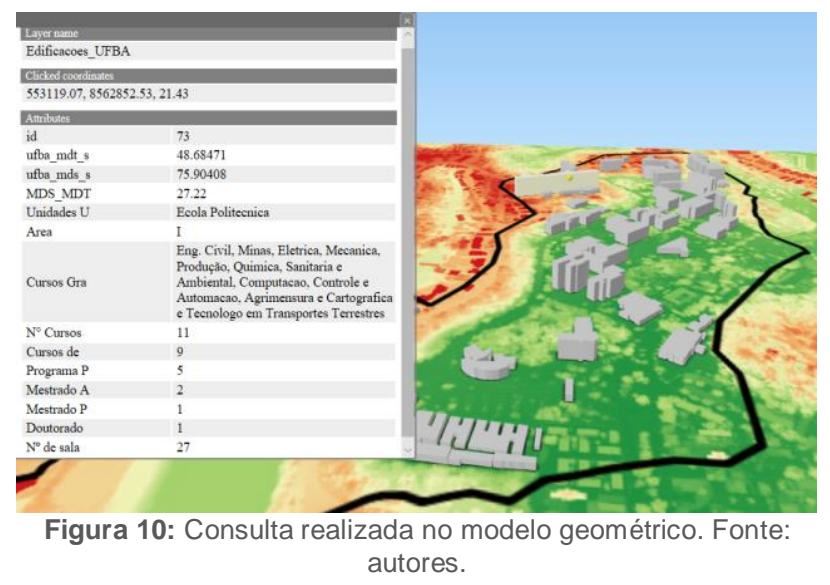


A figura 11 representa a modelagem geométrica a partir do Modelo Digital de Terreno (MDT), considerando as alturas das edificações.

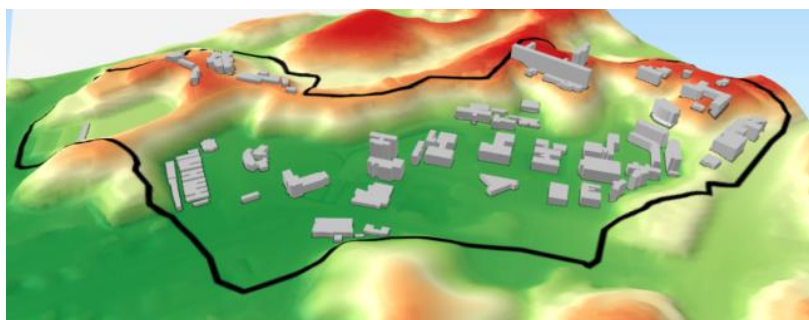

Figura 11: Resultado da modelagem geométrica considerando como base o Modelo Digital de Terreno. Fonte: autores.

\section{CONSIDERAÇÕES FINAIS}

Este trabalho constatou que existem dificuldades no processo de modelagem no que tange a interoperabilidade entre os formatos de arquivo e as diversas plataformas existentes (como foi observado nos exemplos de softwares de modelagem de cidades vistos no item 2.2). Isso significa que principalmente quanto à integração de dados para compor um 3D GIS, a maioria dos softwares de modelagem não importa diretamente arquivos em formato shapefile e/ou não importa/exporta modelos em formato CityGML (padrão atualmente mais utilizado para modelagem geométrica da cidade). No entanto, estudos buscam aperfeiçoar padrões e formatos visando a interoperabilidade de dados para modelagem geométrica.

Considera-se que a modelagem geométrica realizada no campus Ondina-Federação da UFBA representa um estudo em fase inicial em busca de alternativas mais adequadas para a modelagem de edificações utilizando dados provenientes de varredura a laser aerotransportada. Entretanto, comprovou-se que o QGIS mostrou-se eficiente e eficaz para a produção de modelos geométricos simplificados (LOD1) do padrão CityGML. No entanto no QGIS, até este momento da pesquisa, não foi possível exportar o modelo para o formato CityGML, ficando a visualização do modelo restrita ao formato HTML.

Como contribuição, este artigo aplicou procedimentos para a modelagem geométrica para campus universitário, através de dados de varredura a laser aerotransportado, utilizando um complemento de uma ferramenta SIG (2D) para modelagem tridimensional, que permitiu agregar geometria, aparência, semântica e topologia.

Sendo assim, entende-se que este trabalho representa mais um avanço em direção à construção de uma metodologia para modelagem urbana em ambientes 3D GIS. Todavia verificou-se que para modelagem geométrica urbana compatível com os LOD2 e LOD3 do CityGML seria necessário usar ferramentas específicas e plugins como o CityEditor do Sketchup, bem como a disponibilidade de dados mais detalhados provenientes de técnicas como a fotogrametria terrestre.

Pelo fato desta pesquisa encontrar-se em estágio inicial ainda não foi possível testar todas as ferramentas de modelagem de cidades citadas no artigo. Do mesmo modo, a equipe encontrou alguma dificuldade no uso de determinadas ferramentas, notadamente em relação à importação de arquivos em formato shapefile. Estas lacunas representam algumas das metas a serem desenvolvidas na continuidade deste projeto.

\section{REFERÊNCIAS}

AKMALIA, R.; SETAN, H.; MAJID, Z.; SUWARDHI, D. Representing 3D model of building from TLS data scanning in CityGML. Jurnal Teknologi (Sciences \& Engineering). Malaysia: Penerbit UTM Press Universiti Teknologi Malaysia, v. 71 , n. 4 , p. $47-51,2014$.

AMORIM, A. L. Estabelecendo requisitos para a Modelagem da Informação da Cidade (CIM): espaços e fronteiras da Modelagem da Informação da Cidade (CIM). In: Encontro da Associação Nacional de Pesquisa e Pós-Graduação em Arquitetura e Urbanismo, 4., 2016, Porto Alegre. Anais... Porto Alegre: Programa de Pesquisa e Pós-Graduação em Arquitetura e Urbanismo da Universidade Federal do Rio Grande do Sul, 2016

BANSAL, V. K. Use of geographic information systems in spatial planning: a case study of an institute campus. Journal of Computing in Civil Engineering. Fairfax: American Society of Civil Engineers, v. 28, n. 4, 2014

BILJECKI, Filip. Level of detail in 3D city models. 2017. $353 \mathrm{f}$. Tese (Doctor of Philosophy). Technische Universiteit Delft, Delft, 2017.

BOBKOWSKA, K.; SZULWIC, J.; TYSIAC, P.; ZIÓLKOWSKI, P. 2017. GIS three dimensional modelling with geo-informatics techniques. In: International Conference "Environmental Engineering", 10., 2017, Vilnius. Anais... Vilnius: Vilnius Gediminas Technical University, 2017.

CENTENO, J. A. S.; MITISHITA, E. A. Laser scanner aerotransportado no estudo de áreas urbanas: a experiência a UFPR. In: SIMPÓSIO BRASILEIRO DE SENSORIAMENTO REMOTO, 8., 2007, Florianópolis, Anais... São José dos Campos: Instituto Nacional de Pesquisas Espaciais, 2007, p. 3645-3652.

3DISGmbH, CityEditor User Manual, Bocholt, Germany (2016).

ELIAS, ELIAS NASR NAIM. Atualização cartográfica através de imagens obtidas com Veículo Aéreo não Tripulado (VANT) quadrotor. Área de Estudo: Campi da Universidade Federal da Bahia. Trabalho de Conclusão de Curso (Graduação em Engenharia de Agrimensura e Cartográfica) - Escola Politécnica, Universidade Federal da Bahia. Salvador, 2017.

GRÖGER, Gerhard; PLÜMER, Lutz. CityGML - Interoperable semantic 3D city models. Journal of Photogrammetry and Remote Sensing. Amsterdam; New York: Elsevier, v. 71, p. 12-33, jul. 2012.

GRÖGER, Gerhard; KOLBE, Thomas H.; NAGEL, Claus; HÄFELE, Karl-Heinz. OGC City Geography Markup Language (CityGML) encoding standard. Wayland: Open Geospatial Consortium, 2012, 344 p.

JULIN, A.; JAALAMA, K.; VIRTANEN, J. P.; POUKE, M.; YLIPULLI, J.; VAAJA, M.; HYYPPÄ, J.; HYYPPÄ, H. Characterizing 3D City Modeling Projects: Towards a Harmonized Interoperable System. International Journal of Geo-Information. Basel: MDPI AG, v. 7, n. 55, p. 1-,18, fev. 2018.

KEMEC, S.; ZLATANOVA, S.; DUZGUN, S. A framework for defining a $3 D$ model in support of risk management. In: KONECNY, M.; ZLATANOVA, S. BANDROVA, T. Geographic information and cartography for risk and crisis management. New York: Springer, 2010, p. 69-82.

KOEHL, M.; MEYER, E.; KOUSSA, C.; LOTT, C. SIG 3D ET 3D dans les SIG: Application aux modèles patrimoniaux. In: GéoEvénement 2008, Apr 2008, France. p.1-15, 2008.

KUTZNER, T.; KOLBE, T. H. CityGML 3.0: sneak preview. In: Wissenschaftlich-Technische Jahrestagung der DGPF, 38. 2018, Munique. Proceedings... Munique: Deutschen 
Gesellschaft für Photogrammetrie, Fernerkundung und Geoinformation (DGPF), 2018.

LIMA, M. Q. C; FREITAS, F. S. Modelagem paramétrica e os limites dos mecanismos tradicionais de regulação da forma urbana. Revista Políticas Públicas \& Cidades, v.4, n.1, p.117 - 138, jan./jul., 2016.

REDWEIK, P.; TEVES-COSTA, P.; VILAS-BOAS, I.; SANTOS, T. 3D city models as a visual support tool for the analysis of buildings seismic vulnerability: the case of Lisbon. International Journal of Disaster Risk Science. Beijing: Beijing Normal University, v. 8, n. 3, set. 2017.

SILVA JUNIOR, Edgard Rosa. Urbanismo paramétrico: experimentos para uma cidade compacta e sustentável. In: CONGRESS OF THE IBEROAMERICAN SOCIETY OF DIGITAL GRAPHICS, 20., 2016, Buenos Aires. Anais... São Paulo: Associação Ibero-Americana de Gráfica Digital, 2016.
SIRET, D.; MUSY, M.; RAMOS, F.; GROLEAU, D.; JOANNE, P. Développement et mise en oeuvre d'un SIG 3D environnementa lurbain. Revue Internationale de Géomatique. Cachan: Lavoisier SAS, v. 16, n. 1, p. 71-91, 2006.

Universidade Federal da Bahia. Estatuto e regimento geral. Salvador: Universidade Federal da Bahia, 2010.

ZLATANOVA, S.; RAHMAN, A. A.; PILOUK, M. Trends in 3D GIS Development. Journal of Geospatial Engineering. Hong Kong: Hong Kong Institution of Engineering Surveyors, v. 4, n. 2, p. 71-80, dez. 2002.

ZLATANOVA S., STOTER J., ISIKDAG U.: Standards for Exchange and Storage of 3D Information: Challenges and Opportunities for Emergency Response. In Proceedings of the th International Conference on Cartography GIS (Albena, Bulgaria, June 2012), Bandrova T., Konecny M., Zhelezov G.,

(Eds.),

$\mathrm{pp}$.

$17-28$ 